\title{
La formación inicial de los futuros maestros en recursos para la convivencia escolar y el manejo del aula
}

\author{
David Álvarez-García, Celestino Rodríguez, Paloma González-Castro, \\ José C. Núñez y Luís Álvarez \\ Universidad de Oviedo (España)
}

\begin{abstract}
Una de las claves más importantes para prevenir o tratar problemas de convivencia en los centros educativos es conocer instrumentos, técnicas y procedimientos eficaces para ello. En este artículo se pretende analizar en qué medida los futuros maestros de Educación Infantil y Primaria dicen conocer ciertos recursos fundamentales para mantener un buen clima de convivencia escolar, así como su grado de satisfacción con la formación inicial recibida al respecto. Mediante un cuestionario elaborado "ad hoc", fueron evaluados 126 estudiantes de último curso de la Diplomatura de Magisterio de la Universidad de Oviedo. Los resultados revelan un escaso conocimiento de muchos de los recursos evaluados, así como una escasa satisfacción con la formación recibida.
\end{abstract}

Palabras clave: Violencia escolar, formación, profesorado.

Training of pre-service teachers in school coexistence and classroom management resources. One of the most important keys to preventing or treating school coexistence problems is to know effective instruments, techniques and procedures. The aims of this paper are to analyze if pre-service teachers know some of the most important resources to keep a good school climate and, secondly, if they are satisfied with the training that they are receiving at university about these resources. One hundred and twenty six students of the last course of Teacher Training degree at Oviedo University were assessed with an " $a d$ hoc" questionnaire. The results show a poor knowledge of many of the assessed resources and a low satisfaction with received training.

Key words: School violence, training, teachers.

Correspondencia: David Álvarez García. Universidad de Oviedo. Departamento de Psicología. Área de Psicología Evolutiva y de la Educación. Plaza Feijóo, s/n, C.P. 33003 Oviedo (España). E-mail: alvarezgardavid@uniovi.es 
Entre los objetivos del sistema educativo destacan dos de manera especial. Uno es que el alumnado aprenda, como medio para adquirir una cultura suficiente que le permita manejarse en sociedad y promocionar académica y profesionalmente. El otro es que la vida en el centro transcurra en un clima de respeto y cordialidad, a fin de que la comunidad educativa pueda trabajar a gusto para alcanzar los objetivos académicos y, además, se pueda promover en el alumnado un correcto desarrollo social y afectivo.

En los últimos años, se han publicado diversos informes diagnósticos que han tratado de evaluar de forma sistemática el grado de aparición de problemas de convivencia en los centros educativos y que han advertido de su relevancia (ÁlvarezGarcía et al., 2008; Félix, Soriano, Godoy y Sancho, 2010; Gázquez, Pérez-Fuentes, Lucas y Fernández, 2009). Se trata de un problema de origen multicausal, en el que están implicados factores personales, familiares, educativos y sociales (Álvarez, Campo, Castro y Álvarez, 2009; Carbonero, Ortiz, Martín y Valdivieso, 2010; Leiva, 2009; Martorell, González, Rasal y Estellés, 2009; Otero, Castro, Villardefrancos y Santiago, 2009; Raya, Pino y Herruzo, 2009; Sandoval, 2009), por lo que sus vías de solución también deben ser múltiples.

Una de las claves para la prevención y el tratamiento de los problemas de convivencia en los centros educativos es que el profesorado conozca herramientas y procedimientos eficaces para ello. En este sentido, resulta fundamental que el profesorado tenga la posibilidad de recibir una buena formación, tanto inicial como permanente, para el manejo de tales recursos. El presente estudio centrará su atención, en concreto, en la formación inicial de los futuros maestros.

No son muchos los estudios que han tratado de determinar con cierto rigor cuáles son los recursos que conocen y manejan los futuros docentes cuando terminan su preparación obligatoria y en cuáles muestran más carencias. Algunos de estos estudios recaban la opinión de docentes ya en activo sobre la formación universitaria que recibieron (Merrett y Wheldall, 1993). Otros, en la misma línea que el que aquí se presenta, recogen la opinión de los futuros docentes durante sus últimos momentos de formación universitaria.

Un importante trabajo, centrado en el acoso entre estudiantes (Bullying) y que sirvió de referencia para estudios posteriores, es el desarrollado en Inglaterra por Nicolaides, Toda y Smith (2002). En un estudio con estudiantes de grado y postgrado en Educación de las Universidades de Londres y Surrey Roehampton hallaron que, aunque el Bullying se consideraba un tema de gran importancia, la gran mayoría no se sentían confiados en su capacidad para tratar casos de Bullying y consideraban necesaria una mayor formación al respecto.

En Estados Unidos, Bauman y Del Río (2005) replicaron el estudio de Nicolaides et al. (2002) con una muestra de 82 estudiantes universitarios, aspirantes a maestros. Evaluaron, utilizando el cuestionario del estudio inglés, los conocimientos, 
actitudes y creencias sobre el Bullying de estos estudiantes. Obtuvieron unos resultados similares a los del estudio realizado en Inglaterra: ausencia de una clara comprensión de la naturaleza y definición del Bullying; presencia de creencias que pueden dar lugar de hecho a intervenciones inefectivas, cuando no dañinas; e interés en un entrenamiento más extenso para encarar el problema como parte de sus programas de preparación como profesores.

En un estudio más extenso, Kandakai y King (2002) evaluaron a más de 800 estudiantes de seis universidades de Ohio (EE.UU.). Los futuros docentes evaluados consideraron importante prevenir la violencia en la escuela. Sin embargo, un considerable porcentaje de ellos no se sentían confiados en su capacidad para enseñar eficazmente al alumnado estrategias que contribuyesen a prevenir la violencia. La mayoría no había recibido formación en su Universidad para la prevención de la violencia escolar. Los que la habían recibido se mostraban más confiados en poder prevenir y tratar la violencia entre estudiantes.

Ya en España, Benítez, García y Fernández (2006), inspirándose también en el trabajo de Nicolaides et al. (2002), evaluaron a alumnado de la Diplomatura de Magisterio, en las especialidades de Educación Infantil y Educación Primaria, así como al alumnado del curso para la obtención del Certificado de Aptitud Pedagógica (CAP) aspirantes a profesores de Secundaria-, todos ellos de la Universidad de Granada. Se encontraron con un vago conocimiento del maltrato entre iguales, a pesar de que lo consideran un problema de gran importancia, y con su demanda de una mayor formación sobre la prevención y el tratamiento de esta problemática. En un estudio posterior, estos mismos autores (García, Benítez y Fernández, 2007) evaluaron a alumnado de último curso de Magisterio, alumnado del CAP y docentes en activo de Educación Secundaria de centros de la capital de Granada. Hallaron de nuevo una baja autoconfianza por parte de los docentes en formación en su capacidad para afrontar el maltrato entre estudiantes, inferior a la de los docentes en activo. Y encontraron que, en cambio, apenas había diferencias significativas entre los futuros docentes y los docentes en activo respecto a sus demandas de formación para encarar el problema.

Por otro lado, desde la Universidad de Huelva, Alonso, Lobato, Hernando y Cecilia (2007) evaluaron al alumnado de $3^{\circ}$ de Magisterio y del CAP de su Universidad. Entre otros resultados, este alumnado opinó que el profesorado no está suficientemente preparado para abordar los problemas de acoso escolar y que sería necesaria una formación especializada al respecto. La necesidad de formación fue reclamada en mayor medida por el alumnado del curso para la obtención del Certificado de Aptitud Pedagógica (CAP).

En suma, los estudios hasta ahora citados parecen mostrarnos cómo, a pesar de la importancia que los futuros docentes conceden al problema de la violencia escolar, estos presentan carencias en su conocimiento del fenómeno y también de cómo actuar 
sobre él. Muestran, asimismo, una baja competencia percibida en cuanto a su capacidad para abordar los problemas de convivencia. Y demandan una mayor formación en este ámbito. No obstante, estos estudios utilizan muestras locales, por lo que no está justificado que estos resultados puedan generalizarse directamente al alumnado de la Universidad de Oviedo.

El presente trabajo constituye un avance de resultados de una investigación más amplia que se encuentra en curso y que trata de indagar en las posibles carencias formativas que frente a la violencia escolar puedan tener los estudiantes de último curso de la Diplomatura de Magisterio y del curso para la obtención del CAP (futuros maestros y profesores, respectivamente) de la Universidad de Oviedo. Los datos preliminares que se presentan en esta comunicación se refieren específicamente al grado de conocimiento de recursos necesarios para la prevención y el tratamiento de los problemas de convivencia escolar por parte de quienes se están formando para ser maestros de Educación Infantil y Primaria. El objetivo es analizar en qué medida conocen y manejan recursos fundamentales para prevenir y solucionar problemas de convivencia escolar, así como el grado de satisfacción con la formación recibida al respecto.

\section{METODO}

\section{Participantes}

Fueron evaluados 126 estudiantes de tercer curso de la Diplomatura de Magisterio, pertenecientes a la Escuela Universitaria de Magisterio de la Universidad de Oviedo en sus seis especialidades: Educación Infantil, Educación Primaria, Lengua Extranjera, Educación Especial, Educación Física y Educación Musical. La mayoría, un $80.2 \%$, son mujeres y el resto, un $19.8 \%$, son hombres. Sus edades varían entre los 20 y los 44 años. El $89.7 \%$ de la muestra se sitúa entre los 20 y los 25 años. La edad más frecuente son los 20 años (el 33.3\%). La mayoría, un 77\%, carece de experiencia docente al margen de las prácticas de la diplomatura. El $16.7 \%$ tiene un año o menos de experiencia; el $4.8 \%$ tiene entre uno y dos años de experiencia; y el $1.6 \%$ restante tiene más de dos años de experiencia.

\section{Instrumentos}

Se ha utilizado un cuestionario propio, elaborado "ad hoc" para el estudio, en fase de validación. Consta de 31 ítems y se estructura en dos partes. La primera parte contiene ítems referidos a variables clasificatorias. Se pregunta por el género, la edad, los estudios que se cursan en la actualidad, el tiempo de experiencia docente y el grado de satisfacción con la formación que ha recibido sobre convivencia escolar y manejo de la clase. La segunda parte consiste en una escala tipo Likert de 31 enunciados, con cinco opciones de respuesta. Los 31 enunciados se refieren a recursos, técnicas o herramientas que un docente debería saber manejar para abordar o prevenir problemas de convivencia 
escolar. En concreto, se pregunta por el grado de conocimiento de Procedimientos para la detección de problemas de comportamiento y convivencia, Aspectos organizativos (documentos del centro, órganos y profesionales implicados), Legislación, Técnicas conductuales para el manejo del aula, Técnicas basadas en la Educación en Resolución de Conflictos, Conocimiento de metodologías de aula para la integración y Habilidades para el manejo de conflictos. El evaluado debe contestar hasta qué punto conoce cada uno de los recursos presentados. Tanto en estos 31 enunciados como en la pregunta sobre el grado de satisfacción con la formación recibida, las cinco opciones de respuesta son 1 = Nada; 2 = Poco; 3 = Algo; 4 = Bastante; y 5 = Mucho.

\section{Procedimiento}

El trabajo que aquí se presenta forma parte de un estudio más amplio, en fase de realización. Y los datos que se ofrecen suponen un avance de resultados. Para la obtención de los datos que aquí se muestran y analizan, se aplicó el cuestionario al alumnado de $3^{\circ}$ (último curso) de la Diplomatura de Magisterio, durante una clase del mes de marzo del curso 2008/2009. Con ello se pretendió evaluarles lo más avanzado el curso posible, pero antes de que empezasen el Prácticum II, ya que a partir de ese momento se haría muy complicado evaluar al conjunto de la clase de manera colectiva y controlada. Los datos fueron introducidos en el programa SPSS 15.0 para Windows y se realizaron análisis estadísticos en términos de frecuencias y porcentajes.

\section{RESULTADOS}

Conocimiento de procedimientos para la detección de problemas de comportamiento y convivencia

Los resultados obtenidos (Figura 1) muestran que el alumnado de último curso de Magisterio informa tener un escaso conocimiento de instrumentos para la evaluación de la convivencia escolar. Un $63.5 \%$ de los evaluados dice conocer poco o nada cuestionarios de evaluación de la violencia escolar. Un $57.1 \%$ dice conocer poco o nada cuestionarios de evaluación del clima social en el aula. Y un $46.8 \%$ dicen conocer poco o nada procedimientos de registro de conductas observadas en el aula. Los resultados muestran, asimismo, que existe un conocimiento general sólo moderado sobre cómo detectar posibles casos de acoso escolar (Bullying) y algo mayor sobre cómo detectar posibles trastornos de comportamiento.

Conocimiento de aspectos organizativos. Documentos del centro

El alumnado evaluado muestra un escaso conocimiento de algunos de los documentos de centro relacionados con la convivencia escolar (Figura 2). En general, existe un escaso conocimiento de qué es y qué ha de contener el Plan de Acogida de un centro (un $61.9 \%$ dice conocerlo poco o nada y sólo un $9.5 \%$ bastante o mucho), qué es y 
qué ha de contener el Plan Integral de Convivencia (un $56.3 \%$ dice conocerlo poco o nada y sólo un $14.3 \%$ bastante o mucho) y las posibilidades preventivas y de intervención desde el Plan de Acción Tutorial (un 55.6\% dice conocerlo poco o nada y sólo un $12.7 \%$ bastante o mucho). De los cuatro documentos evaluados, el Reglamento de Régimen Interior es, con diferencia, el más conocido. Un $57.2 \%$ dice conocerlo bastante o mucho y sólo el $18.3 \%$ poco o nada.

Figura 1. Grado de conocimiento de PRCOA, CEVE, CECSA, DAE y DTC

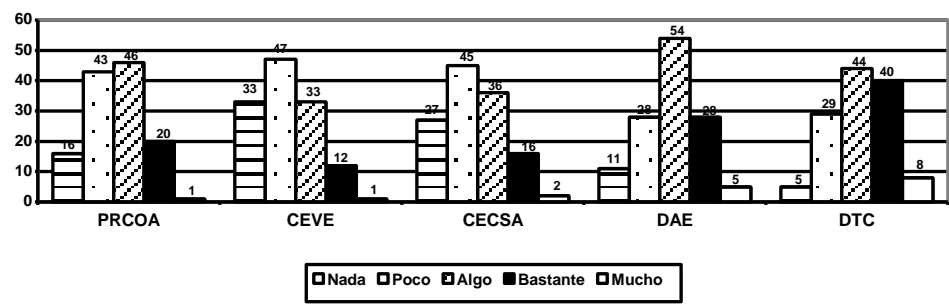

Nota: $(\mathrm{PRCOA}=$ Procedimientos de Registro de Conductas Observadas en el Aula; CEVE $=$ Cuestionarios de Evaluación de la Violencia Escolar; CECSA = Cuestionarios de Evaluación del Clima Social en el Aula) y sobre cómo detectar posibles casos de acoso escolar (DAE) y de trastornos del comportamiento (DTC) declarado por el alumnado de último curso de Magisterio $(\mathrm{N}=126)$. Los dígitos sobre cada columna informan del número de evaluados que han elegido cada opción.

Figura 2. Grado de conocimiento informado por el alumnado sobre PIC, PA, RRI y PAT

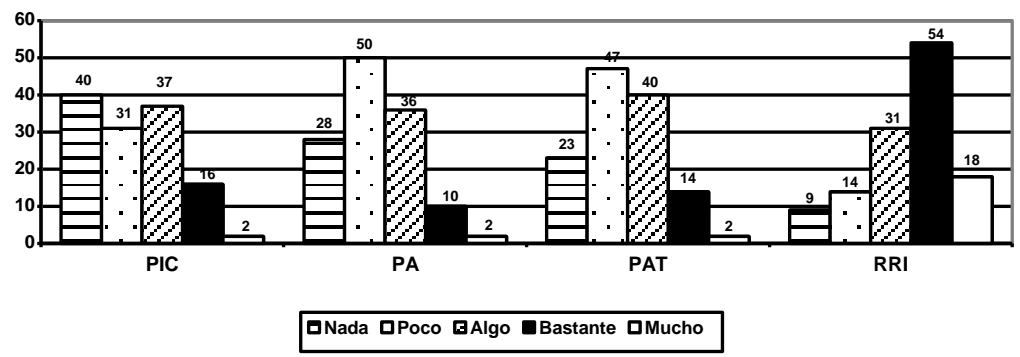

Nota: qué es y qué debe contener el Plan Integral de Convivencia (PIC); el Plan de Acogida de un centro (PA); y el Reglamento de Régimen Interno (RRI); así como sobre las posibilidades preventivas y de intervención desde el Plan de Acción Tutorial (PAT). Los dígitos sobre cada columna informan del número de evaluados que han elegido cada opción.

Conocimiento de aspectos organizativos. Órganos y profesionales implicados

A excepción del Departamento de Orientación, los evaluados apenas conocen el papel de los diferentes órganos y profesionales implicados en la convivencia escolar por los que se preguntó en el cuestionario (Figura 3). Un 67.5\% de los evaluados dice conocer poco o nada las funciones del técnico especialista en Servicios a la Comunidad. Un $62.7 \%$ dice conocer poco o nada el papel de la Inspección Educativa ante casos de 
violencia escolar. Un $61.1 \%$ dice conocer poco o nada colectivos del entorno que trabajen en la prevención de la violencia escolar. Y un $49.2 \%$ dicen conocer poco o nada la composición y funciones de la Comisión de Convivencia. En cambio, el porcentaje de evaluados que conocen poco o nada las funciones de un Departamento de Orientación es bastante menor (el 22.2\%).

Figura 3. Grado de conocimiento informado por el alumnado sobre SC, DO, CC, CE y IE

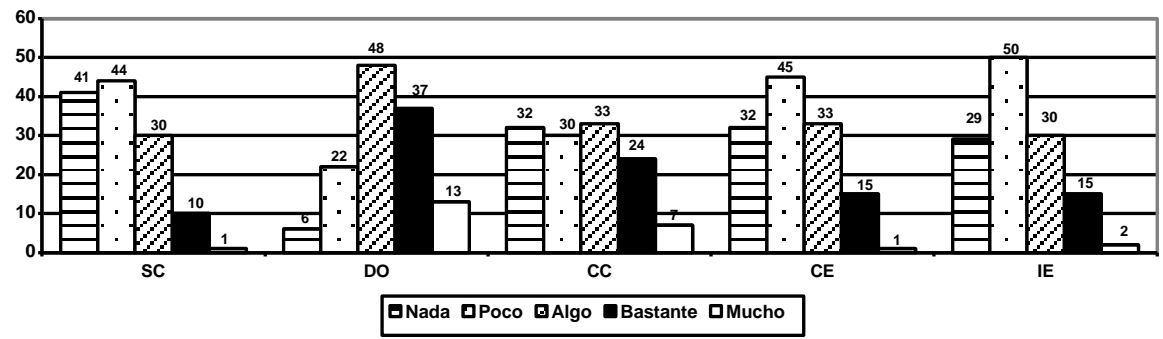

Nota: Las funciones del técnico especialista en Servicios a la Comunidad (SC); las funciones del Departamento de Orientación (DO); la composición y funciones de la Comisión de Convivencia (CC); colectivos del entorno que trabajen en la prevención de la violencia escolar (CE); y el papel de la Inspección Educativa ante casos de violencia escolar (IE). Los dígitos sobre cada columna informan del número de evaluados que han elegido cada opción

Figura 4. Grado de conocimiento informado por el alumnado acerca del Decreto 249/2007 de Asturias, Decreto 732/1995 MEC y Derecho Penal de Menores

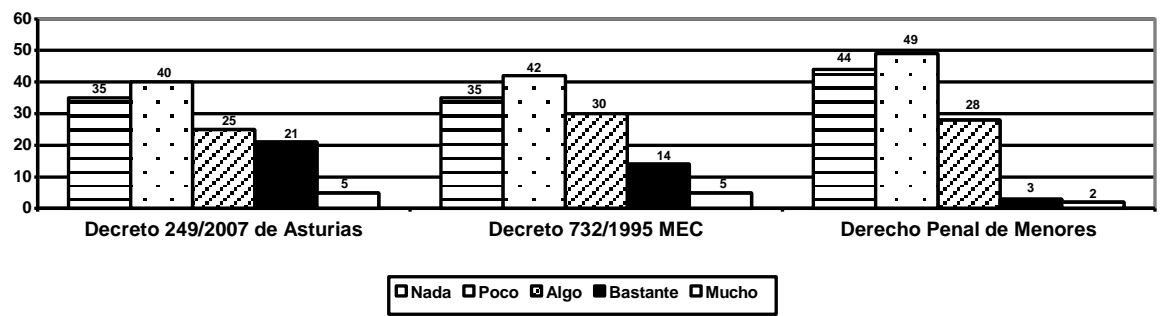

Nota: Decreto 249/2007, por el que se regulan los derechos y deberes del alumnado y normas de convivencia en los centros docentes no universitarios de Asturias; del Real Decreto 732/1995, por el que se establecen los derechos y deberes de los alumnos y las normas de convivencia de los centros en todo el territorio gestionado por el MEC; y del Derecho Penal de Menores ante casos de violencia escolar. Los dígitos sobre cada columna indican el número de evaluados que han elegido cada opción.

\section{Conocimiento de legislación}

Como se advierte en la figura 4, los evaluados muestran un escaso conocimiento legislativo en relación con la convivencia escolar. El 59.5\% informa conocer poco o nada el Decreto 249/2007, por el que se regulan los derechos y deberes del alumnado y normas de convivencia en los centros docentes no universitarios de 
Asturias. El 61.1\% informa conocer poco o nada el Real Decreto 732/1995, por el que se establecen los derechos y deberes de los alumnos y las normas de convivencia de los centros en todo el territorio gestionado por el MEC. El 73.8\% confiesa saber poco o nada acerca del Derecho Penal de menores ante casos de violencia escolar.

Conocimiento de técnicas conductuales para el manejo del aula

El $61.2 \%$ de los evaluados conoce bastante o mucho qué es un reforzador y cómo debe ser aplicado. Un $65 \%$ conoce bastante o mucho la técnica de la economía de fichas. En cambio, el contrato conductual y la técnica "Time-Out" ("Tiempo-Fuera") son menos conocidas (Figura 5).

Figura 5. Grado de conocimiento informado por el alumnado acerca de TO, EF, CC y R

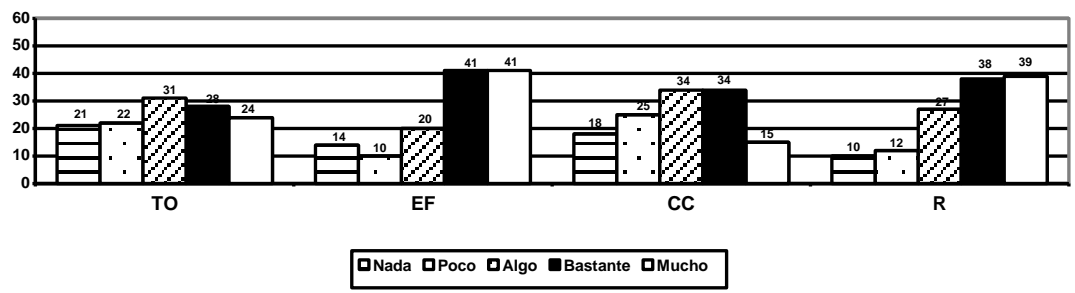

Nota: La técnica "Time-Out" (TO); la economía de fichas (EF); el contrato conductual (CC); y qué es un reforzador y cómo debe ser aplicado (R). Los dígitos sobre cada columna informan del número de evaluados que han elegido cada opción

Figura 6. Grado de conocimiento informado por el alumnado acerca de AA, CN, ME y AY

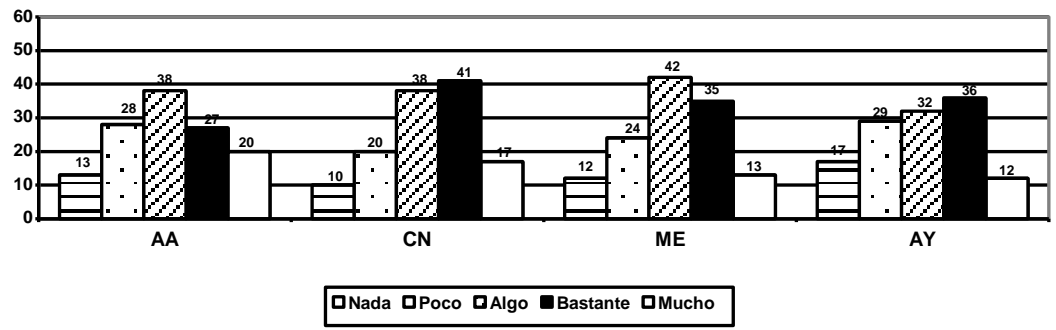

Nota: La asamblea de aula (AA), el consenso de normas $(\mathrm{CN})$, la mediación escolar (ME) y los programas de "alumnos ayudantes" (AY). Los dígitos sobre cada columna informan del número de evaluados que han elegido cada opción.

\section{Conocimiento de técnicas basadas en la Educación en Resolución de}

\section{Conflictos}

El nivel de conocimiento de este tipo de técnicas por parte del alumnado evaluado es muy variado (Figura 6). Si bien el $46 \%$ dice conocer bastante o mucho el consenso de normas, casi una cuarta parte (el 23.8\%) dice conocerlo poco o nada. Del mismo modo, el $38.1 \%$ dice conocer bastante o mucho la mediación escolar, pero más de 
la cuarta parte (un 28.6\%) reconoce conocer esta técnica poco o nada. Respecto a la asamblea de aula y los programas de "alumnos ayudantes", el porcentaje de evaluados que dicen conocerlos es muy similar al que dice desconocerlos.

Figura 7. Grado de conocimiento informado por el alumnado acerca de las técnicas de aprendizaje cooperativo; la técnica "Jigsaw"; y dinámicas de grupo

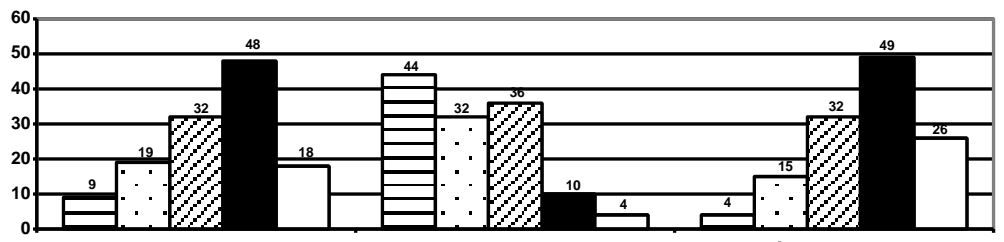

Aprendizaje cooperativo

Jigsaw

Dinámicas de grupo

QNada DPoco ఐAlgo DBastante QMucho

Figura 8. Grado de conocimiento informado por el alumnado sobre HC, HAE y HRC

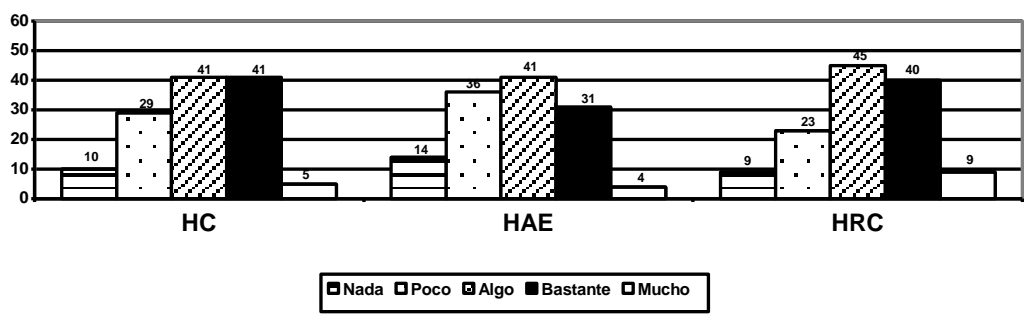

Nota: Habilidades de comunicación (HC); habilidades de autocontrol emocional (HAE); y habilidades de resolución de conflictos a través de la negociación o la mediación (HRC). Los dígitos sobre cada columna informan del número de evaluados que han elegido cada opción

\section{Conocimiento de metodologías de aula para la integración}

Los resultados obtenidos (Figura 7) muestran que tanto las dinámicas de grupo como el aprendizaje cooperativo son técnicas en general conocidas por el alumnado. Sin embargo, cuando se les pregunta por una técnica concreta de aprendizaje cooperativo como es el "rompecabezas" ("jigsaw"), el grado de conocimiento es muy escaso.

\section{Conocimiento de habilidades para el manejo de conflictos}

Más de una cuarta parte del alumnado dice conocer poco o nada habilidades de comunicación, de autocontrol emocional o de resolución de conflictos a través de la negociación o de la mediación (Figura 8). En concreto, un $31 \%$, un $39.7 \%$ y un $25.4 \%$, respectivamente. Existen, no obstante, porcentajes similares -en dos de los casos, superiores- de alumnado que informa conocer bastante o mucho estas habilidades $(36.5 \%, 27.8 \%$ y $38.8 \%$, respectivamente). 
Satisfacción con la formación recibida sobre convivencia escolar y manejo de la clase

El alumnado evaluado se muestra en su mayoría poco satisfecho con la formación recibida (Figura 9). Un 35.7\% se muestra poco satisfecho. Un $28.6 \%$ se muestra nada satisfecho. Un $20.6 \%$ se muestra algo satisfecho. Un $12.7 \%$ se muestra bastante satisfecho. Y sólo un $2.4 \%$ se muestra muy satisfecho.

Figura 9. Grado de satisfacción con la formación recibida sobre convivencia escolar y manejo de la clase informado por el alumnado de último curso de Magisterio $(\mathrm{N}=126)$

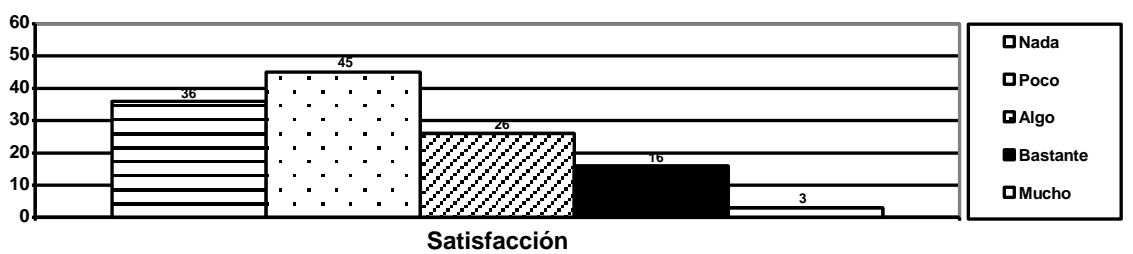

DISCUSION

La principal conclusión de este estudio va en la misma línea que las obtenidas por los estudios revisados (Alonso et al., 2007; Bauman y Del Río, 2005; Benítez et al., 2006; García et al., 2007; Kandakai y King, 2002; Merret y Wheldall, 1993; y Nicolaides et al., 2002). Sería necesaria una mejor formación de las futuras maestras y los futuros maestros en lo referido a la convivencia escolar. Y esta conclusión se extrae tanto de los resultados obtenidos al preguntar al alumnado por su grado de conocimiento de recursos para la convivencia escolar como de los obtenidos al preguntar por su grado de satisfacción con la formación recibida al respecto.

Los resultados obtenidos muestran un conocimiento en general pobre de recursos para la prevención y el tratamiento de los problemas de convivencia escolar. Los futuros maestros reconocieron desconocer especialmente instrumentos para la evaluación de la convivencia escolar, legislación educativa sobre convivencia escolar y ciertos elementos de la organización escolar implicados en la convivencia. En cuanto a esto último, el alumnado evaluado informo tener un escaso conocimiento de documentos del centro como el Plan Integral de Convivencia, el Plan de Acogida o el Plan de Acción Tutorial. Sólo el Reglamento de Régimen Interior es bastante más conocido. El alumnado informó tener, asimismo, un escaso conocimiento de órganos y profesionales implicados en la convivencia escolar como el técnico especialista en Servicios a la Comunidad, la Comisión de Convivencia, la Inspección educativa o colectivos del entorno que trabajan en la prevención de la violencia escolar. Sólo el Departamento de Orientación es bastante más conocido, a pesar de que son pocos los centros de Educación Primaria en Asturias que cuentan con este recurso. 
Hay otros recursos para la convivencia escolar ante los que los futuros maestros evaluados muestran un mayor conocimiento que hacia los anteriores, siendo, sin embargo, aún bastante mejorable. Entre ellos, se encuentran cómo detectar situaciones de acoso escolar (Bullying) y posibles trastornos de comportamiento; ciertas técnicas conductuales, como son el contrato conductual y el "Time-Out"; técnicas basadas en la Educación en Resolución de Conflictos, como la asamblea de aula, el consenso de normas, la mediación escolar o los programas de "alumnos ayudantes"; y habilidades para el manejo de conflictos, como las de comunicación, de autocontrol emocional y de resolución de conflictos a través de la negociación o de la mediación.

Por último, hay ciertos recursos para la convivencia escolar y el manejo de la clase que el alumnado evaluado dice conocer especialmente. Sobre todo, dice conocer ciertas técnicas conductuales, como son el uso adecuado de reforzadores y la economía de fichas, así como dinámicas de grupo y el aprendizaje cooperativo. Respecto a este último, el alumnado dice conocer este tipo de técnicas en general, pero cuando se les pregunta por una técnica concreta como es el "rompecabezas" o "jigsaw" (Aronson y Patnoe, 1997) informan en su gran mayoría desconocerla.

Además del escaso conocimiento acerca de la mayoría de los recursos para la convivencia escolar evaluados, el otro motivo por el cual se llega a la conclusión de que es necesario revisar la formación del futuro profesorado para la convivencia escolar es el bajo nivel de satisfacción informada por los evaluados con la formación recibida al respecto.

En suma, los pobres resultados obtenidos nos indican, en la línea de los estudios revisados (Alonso et al., 2007; Bauman y Del Río, 2005; Benítez et al., 2006; García et al., 2007; Kandakai y King, 2002; Merret y Wheldall, 1993; y Nicolaides et al., 2002), la pertinencia de una más extensa y profunda formación general de los futuros maestros en relación con toda una serie de herramientas útiles para mantener un buen clima de convivencia y de trabajo en el centro educativo.

\section{Agradecimientos}

Esta investigación se ha elaborado en el marco del proyecto de investigación "La formación de los futuros docentes frente a la violencia escolar" (Ref. SV-UNOV-09MB-1), subvencionado por la Universidad de Oviedo.

\section{REFERENCIAS}

Alonso, P., Lobato, H., Hernando, A. y Cecilia, M.V. (2007). La visión del acoso escolar en futuros profesores de Primaria y de Secundaria. En J.J. Gázquez, M.C. Pérez, A.J. Cangas y N. Yuste (Coords.), Mejora de la convivencia y programas encaminados a la prevención e intervención del acoso escolar (pp. 105-110). Granada: Grupo Editorial Universitario. 
Álvarez, E., Campo, M.A., Castro, P. y Álvarez, M. (2009). Visión de los especialistas en pedagogía terapéutica de la integración del alumnado inmigrante. Aula Abierta, 37(1), 57-66.

Álvarez-García, D., Álvarez, L., Núñez, J.C., González-Pienda, J.A., González-Castro, P. у Rodríguez, C. (2008). Estudio del nivel de violencia escolar en siete centros asturianos de Educación Secundaria. Aula Abierta, 36, 89-96.

Aronson, E. y Patnoe, S. (1997). The jigsaw classroom: Building cooperation in the classroom (2nd ed.). New York: Addison Wesley Longman.

Bauman, S. y del Río, A. (2005). Knowledge and beliefs about Bullying in schools: comparing pre-service teachers in United States and the United Kingdom. School Psychology International, 26(4), 428-442.

Benítez, J.L., García, A.B. y Fernández, M. (2006). El maltrato entre alumnos: conocimientos, percepciones y actitudes de los futuros docentes. Revista de Investigación Educativa, 24(2), 329-352.

Carbonero, M.A., Ortiz, E., Martín, L.J. y Valdivieso, J.A. (2010). Identificación de las variables docentes moduladoras del profesor eficaz en Secundaria. Aula Abierta, 38(1), 15-24.

Félix, V., Soriano, M., Godoy, C. y Sancho, S. (2010). El ciberacoso en la enseñanza obligatoria. Aula Abierta, 38(1), 47-58.

García, A.B., Benítez, J.L. y Fernández, M. (2007). La autoeficacia y las demandas de formación del profesorado en activo vs. en formación para afrontar el Bullying. En J.J. Gázquez, M.C. Pérez, A.J. Cangas y N. Yuste (Coords.), Mejora de la convivencia y programas encaminados a la prevención e intervención del acoso escolar (pp. 119-125). Granada: Grupo Editorial Universitario.

Gázquez, J.J., Pérez-Fuentes, M.C., Lucas, F. y Fernández, M. (2009). Análisis de la convivencia escolar por los docentes europeos. Aula Abierta, 37(2), 11-18.

Kandakai, T.L. y King, K.A. (2002). Preservice Teachers' Perceived Confidence in Teaching School Violence Prevention. American Journal of Health Behavior, 26(5), 342-353.

Leiva, J.J. (2009). El profesorado ante la concepción de los conflictos en contextos de educación intercultural. Aula Abierta, 37(2), 93-110.

Martorell, C., González, R., Rasal, P. y Estellés, R. (2009). Convivencia e inteligencia emocional en niños en edad escolar. European Journal of Education and Psychology, 2(1), 69-78.

Merret, F. y Wheldall, K. (1993). How do teachers learn to manage classroom behaviour? A study of teachers' opinions about their initial training with special reference to classroom behaviour management. Educational Studies, 19(1), 91-106.

Nicolaides, S., Toda, Y. y Smith, P.K. (2002). Knowledge and attitudes about school Bullying in trainee teachers. British Journal of Educational Psychology, 72, 105-118.

Otero, J.M., Castro, C., Villardefrancos, E. y Santiago, M.J. (2009). Job dissatisfaction and burnout in secondary school teachers: student's disruptive behaviour and conflict management examined. European Journal of Education and Psychology, 2(2), 99-111.

Raya, A.F., Pino, M.J. y Herruzo, J. (2009). La agresividad en la infancia: el estilo de crianza parental como factor relacionado. European Journal of Education and Psychology, 2(3), 211-222.

Sandoval, M. (2009). Concepciones de los estudiantes de magisterio sobre la inclusión educativa. Aula Abierta, 37(1), 79-88.

Recibido: 14 de enero de 2010

Recepción Modificaciones: 19 de marzo de 2010

Aceptado: 24 de marzo de 2010 